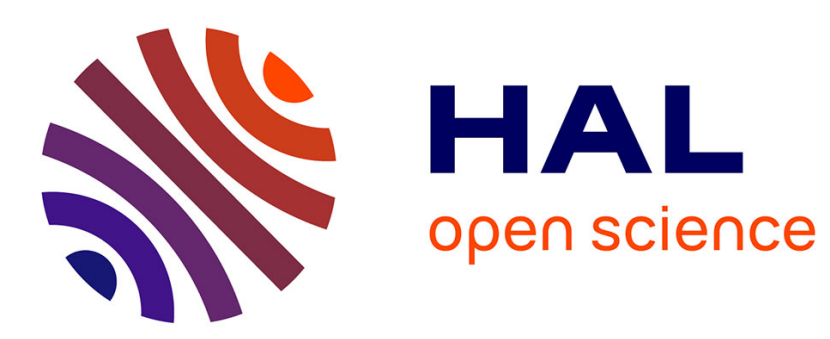

\title{
Generalized zeon algebras: theory and application to multi-constrained path problems
}

\author{
René Schott, George Stacey Staples
}

\section{To cite this version:}

René Schott, George Stacey Staples. Generalized zeon algebras: theory and application to multi-constrained path problems. Advances in Applied Clifford Algebras, 2017, 27 (1), pp.45-57. 10.1007/s00006-015-0595-0 . hal-00603748

\section{HAL Id: hal-00603748 https://hal.science/hal-00603748}

Submitted on 27 Jun 2011

HAL is a multi-disciplinary open access archive for the deposit and dissemination of scientific research documents, whether they are published or not. The documents may come from teaching and research institutions in France or abroad, or from public or private research centers.
L'archive ouverte pluridisciplinaire HAL, est destinée au dépôt et à la diffusion de documents scientifiques de niveau recherche, publiés ou non, émanant des établissements d'enseignement et de recherche français ou étrangers, des laboratoires publics ou privés. 


\title{
Operator calculus in generalized zeon algebras: theory and application to multi-constrained path problems
}

\author{
René Schott, G. Stacey Staples ${ }^{\dagger}$
}

\begin{abstract}
Classical approaches to routing problems invariably require construction of trees and the use of heuristics to prevent combinatorial explosion. The operator calculus approach presented herein, however, allows such explicit tree constructions to be avoided. Introduced here is the notion of generalized zeon algebras and their associated operator calculus. The inherent combinatorial properties of generalized zeons make them useful for routing problems by implicitly pruning the underlying tree structures. As an application, an operator calculus approach to multi-constrained path problems is described.

keywords: shortest paths, message routing, operator calculus, semigroup algebras

MSC: 68W30, 81R05
\end{abstract}

\section{Introduction}

Operator calculus (OC) methods on graphs have been developed in a number of earlier works by Schott and Staples [6, 7, 8, 9]. The principal idea underlying the approach is the association of graphs with algebraic structures whose properties reveal information about the associated graphs. By constructing the "nilpotent adjacency matrix" associated with a finite graph, information about self-avoiding structures (paths, cycles, trails, etc.) in the graph are revealed by computing powers of the matrix.

In the operator calculus approach, graded semigroup algebras are generated by "null-square" elements such that properties of the algebra "sieve out" paths. In other words, cycles are removed from consideration automatically.

Consider a directed graph $G=(V, E)$ on $n$ vertices such that associated with each edge $\left(v_{i}, v_{j}\right) \in E$ is a vector weight $\mathbf{w}_{i j}=\left(w_{i j 1}, \ldots, w_{i j m}\right) \in \mathbb{R}^{m}$.

${ }^{*}$ IECN and LORIA, Nancy Université, Université Henri Poincaré, BP 239, 54506 Vandoeuvre-lès-Nancy, France, Email: schott@loria.fr

${ }^{\dagger}$ Corresponding author. Department of Mathematics and Statistics, Southern Illinois University Edwardsville, Edwardsville, IL 62026-1653, Email: sstaple@siue.edu 
The point $w^{*}=\left(w_{1}^{*}, \ldots, w_{m}^{*}\right) \in X \subset \mathbb{R}^{m}$ is referred to as a Pareto minimum of $X$ if there does not exist $\mathbf{w}=\left(w_{1}, \ldots, w_{m}\right) \in X$ such that

$$
\begin{gathered}
(\forall i)\left[w_{i} \leq w_{i}^{*}\right] \text {, and } \\
(\exists j)\left[w_{j}<w_{j}^{*}\right] .
\end{gathered}
$$

Equivalently, one says that $w^{*}$ is nondominated from below.

Defining the weight of a path in an edge-weighted graph as the sum of vector weights of arcs contained in the path, a Pareto path is then a path whose weight is a Pareto minimum.

For the case $m=1$, Dijkstra's algorithm finds all single source minimum paths in a directed graph on $n$ vertices with nonnegative edge weights in $\mathcal{O}\left(n^{2}\right)$ time [4]. The Bellman-Ford algorithm finds single source minimal paths in digraphs with arbitrary edge weights and runs in $\mathcal{O}(n|E|)$ time $[1,5]$.

In the more general case $m \geq 1$, Corley and Moon [2] presented an algorithm for finding all Pareto paths with computational complexity $\mathcal{O}\left(m n^{2 n-3}+m n^{n}\right)$.

The aim of the current work is to find Pareto paths satisfying multiple constraints. Given a vector $\mathbf{c}=\left(c_{1}, \ldots, c_{m}\right) \in \mathbb{R}^{m}$, a path is deemed feasible if its vector weight $\mathbf{w}=\left(w_{1}, \ldots, w_{m}\right)$ satisfies

$$
(\forall i)\left[w_{i} \leq c_{i}\right]
$$

Letting $\mathcal{P}_{f}$ denote the collection of feasible paths having fixed source $v_{0}$ and fixed target $v_{\infty}$, the goal is to find a path in $\mathcal{P}_{f}$ whose weight is a Pareto minimum. The operator calculus approach described herein can be applied to sieve out the collection of feasible paths and recover all single-source Pareto paths remaining.

\section{Theory: Generalized zeon algebras}

Zeon algebras are commutative algebras generated by collections of null-squares, $\left\{\zeta_{i}: 1 \leq i \leq n\right\}$ with $\zeta_{i}{ }^{2}=0$ for each $i$. Their combinatorial properties make them useful for a variety of counting properties, as seen in a number of previous works by the current authors. By choosing sufficiently large sets of generators, they can be generalized to algebras whose generators are nilpotent of arbitrary index. The resulting generalized zeon algebras are suitable for a number of combinatorial applications, including multi-constrained routing problems.

Definition 2.1. The $n$-particle zeon algebra, denoted by $\mathcal{C} \ell_{n}{ }^{\text {nil }}$, is defined as the real abelian algebra generated by the collection $\left\{\zeta_{i}\right\}(1 \leq i \leq n)$ along with the scalar $1=\zeta_{0}$ subject to the following multiplication rules:

$$
\begin{gathered}
\zeta_{i} \zeta_{j}=\zeta_{j} \zeta_{i} \text { for } i \neq j, \text { and } \\
\zeta_{i}{ }^{2}=0 \text { for } 1 \leq i \leq n
\end{gathered}
$$


A general element $u \in \mathcal{C} \ell_{n}{ }^{n i l}$ can be expanded as

$$
u=\sum_{I \in 2^{[n]}} u_{I} \zeta_{I}
$$

where $I \in 2^{[n]}$ is a subset of $[n]=\{1,2, \ldots, n\}$ used as a multi-index, $u_{I} \in \mathbb{R}$, and $\zeta_{I}=\prod_{\iota \in I} \zeta_{\iota}$.

Remark 2.2. The zeon algebra $\mathcal{C} \ell_{n}{ }^{\text {nil }}$ can be realized as a commutative subalgebra of the Grassmann algebra $\bigwedge V$ over a $2 n$-dimensional vector space $V$ with orthonormal basis $\left\{\gamma_{i}\right\}$ by defining $\zeta_{i}=\gamma_{i} \gamma_{n+i}$ for each $1 \leq i \leq n$.

A canonical basis element $\zeta_{I}$ is referred to as a blade. The number of elements in the multi-index $I$ is referred to as the grade of the blade $\zeta_{I}$.

The next lemma shows that it is possible to construct elements with arbitrary index of nilpotency within a zeon algebra of sufficiently high dimension.

Lemma 2.3. Let $\left\{\zeta_{i}: 1 \leq i \leq n\right\}$ be the null-square generators of $\mathcal{C} \ell_{n}{ }^{\text {nil }}$. Then, for any permutation $\sigma \in S_{n}$ and positive integers $\ell \leq k \leq n$,

$$
\left(\sum_{j=1}^{k} \zeta_{\sigma(j)}\right)^{\ell}=\ell ! \sum_{\substack{I \subseteq\{\sigma(1), \ldots, \sigma(k)\} \\|I|=\ell}} \zeta_{I} .
$$

Moreover, if $\ell>k$, then

$$
\left(\sum_{j=1}^{k} \zeta_{\sigma(j)}\right)^{\ell}=0 .
$$

Proof. Since the generators commute, the multinomial theorem applies, with only square-free terms surviving.

Definition 2.4. For positive integer $n$, let $\mathbf{s}=\left(s_{1}, \ldots, s_{n}\right) \in \mathbb{N}^{n}$ be an $n$-tuple of positive integers. Then, the zeon algebra of signature $\mathbf{s}$ (or $\mathbf{s}$-zeon algebra), denoted $\mathcal{C} \ell_{\mathbf{s}}{ }^{\text {nil }}$, is the real abelian algebra generated by the collection $\left\{\nu_{i}\right\} \quad(1 \leq$ $i \leq n$ ) along with the scalar $1=\nu_{0}$ subject to the following multiplication rules:

$$
\begin{gathered}
\nu_{i} \nu_{j}=\nu_{j} \nu_{i} \text { for } i \neq j, \text { and } \\
\nu_{i}{ }^{s_{i}}=0 \text { for } 1 \leq i \leq n .
\end{gathered}
$$

For convenience, the following multi-exponent notation is adopted:

$$
\nu^{\mathbf{x}}:=\prod_{i=1}^{n} \nu_{i}^{x_{i}}:=\nu_{1}^{x_{1}} \cdots \nu_{n}^{x_{n}}
$$

Letting $\mathcal{S}=\left\{\left(x_{1}, \ldots, x_{n}\right): 0 \leq x_{i} \leq s_{i}\right\} \subset \mathbb{N}_{0}{ }^{n}$, a general element $u \in \mathcal{C} \ell_{\mathbf{s}}{ }^{\text {nil }}$ can be expanded as

$$
u=\sum_{\mathbf{x} \in \mathcal{S}} u_{\mathbf{x}} \nu^{\mathbf{x}}
$$

where $u_{\mathbf{x}} \in \mathbb{R}$ for each multi-exponent $\mathbf{x}$. 
Since the components of signature vectors and arbitrary multi-exponents are nonnegative integers, the 1-norm of such a vector $\mathbf{x}$ is simply the sum of the components; that is,

$$
\|\mathbf{x}\|_{1}=\sum_{i=1}^{n}\left|x_{i}\right|=\sum_{i=1}^{n} x_{i}
$$

Lemma 2.5. The algebra $\mathcal{C} \ell_{\mathbf{s}}{ }^{\text {nil }}$ is isomorphic to a subalgebra of the zeon algebra $\mathcal{C} \ell_{\|\mathbf{s}\|_{1}}{ }^{\text {nil }}$.

Proof. For $k \in\{1, \ldots, n\}$, let $p(k)$ denote the $k^{\text {th }}$ partial sum

$$
p(k)=\sum_{j=1}^{k} s_{j}
$$

and define $p(0)=0$. In light of Lemma 2.3 , the desired isomorphism $\mathcal{C} \ell_{\mathbf{s}}{ }^{\text {nil }} \rightarrow$ $\mathcal{C} \ell_{\|\mathbf{s}\|_{1}}{ }^{\text {nil }}$ is obtained from the mapping

$$
\nu_{i} \mapsto \sum_{j=1}^{s_{i}} \zeta_{j+p(i-1)}
$$

Finally, note that the s-zeon algebra is naturally graded according to

$$
\mathcal{C} \ell_{\mathbf{s}}{ }^{\text {nil }}=\bigoplus_{k=0}^{\|\mathbf{s}\|_{1}}\left\langle\mathcal{C} \ell_{\mathbf{s}}{ }^{n i l}\right\rangle_{k}
$$

where the grade- $k$ part of the algebra is defined by

$$
\left\langle\mathcal{C} \ell_{\mathbf{s}}{ }^{\text {nil }}\right\rangle_{k}=\operatorname{span}\left(\left\{\nu^{\mathbf{x}}:\|\mathbf{x}\|_{1}=k\right\}\right) .
$$

The notation $\langle\cdot\rangle_{k}$ extends naturally to elements of $\mathcal{C} \ell_{\mathbf{s}}{ }^{\text {nil }}$.

\section{Operator calculus in generalized zeon algebras}

The motivation for development of s-zeon operator calculus is based on polynomial operator calculus. To begin, raising and lowering operators are defined naturally in terms of polynomial differentiation and integration operators on basis zeons regarded as polynomials in commuting variables. In this formulation, the generators $\left\{\nu_{i}\right\}$ of $\mathcal{C} \ell_{\mathbf{s}}{ }^{\text {nil }}$ are regarded as variables in the polynomial sense.

For any generalized zeon algebra with $n$ generators, let $\left\{\mathbf{e}_{i}: 1 \leq i \leq n\right\}$ denote standard unit vectors of the form $\mathbf{e}_{i}:=(0, \ldots, \underbrace{1}_{i^{\text {th }} \text { pos. }}, \ldots, 0)$. Arbitrary

multi-exponents are then expressed in the form $\mathbf{x}=\left(x_{1}, \ldots, x_{n}\right)=\sum_{i=1}^{n} x_{i} \mathbf{e}_{i}$. 
Definition 3.1. Let $\mathbf{s} \in \mathbb{N}_{0}{ }^{n}$ be an arbitrary zeon signature. For $1 \leq j \leq n$, define the $j^{\text {th }} \mathbf{s}$-zeon differentiation operator $\partial / \partial \nu_{j}$ on $\mathcal{C} \ell_{\mathbf{s}}{ }^{\text {nil }}$ by linear extension of

$$
\frac{\partial}{\partial \nu_{j}} \nu^{\mathbf{x}}= \begin{cases}\nu^{\mathbf{x}-\mathbf{e}_{j}} & \text { if } x_{j} \geq 1, \\ 0 & \text { otherwise. }\end{cases}
$$

Definition 3.2. The s-zeon integrals are defined by

$$
\int \nu^{\mathbf{x}} \mathrm{d} \nu_{j}= \begin{cases}\nu^{\mathbf{x}+\mathbf{e}_{j}} & \text { if } x_{j}<s_{j}-1, \\ 0 & \text { otherwise. }\end{cases}
$$

These polynomial operators induce combinatorial raising and lowering operators by which s-zeon monomials (blades) are raised from grade $k$ to grade $k+1$ or lowered from grade $k$ to grade $k-1$. These raising and lowering operators can also be regarded as creation and annihilation operators in the sense of quantum mechanics.

Definition 3.3. For each $1 \leq j \leq n$, define the $j^{\text {th }}$ raising operator $R_{j}$ by linear extension of

$$
R_{j} \nu^{\mathbf{x}}=\int \nu^{\mathbf{x}} \mathrm{d} \nu_{j}=\nu^{\mathbf{x}} \nu_{j}
$$

Define the $j^{\text {th }}$ lowering operator $D_{j}$ by linear extension of

$$
D_{j} \nu^{\mathbf{x}}=\frac{\partial}{\partial \nu_{j}} \nu^{\mathbf{x}}
$$

Definition 3.4. The $j^{\text {th }}$ zeon number operator $\Lambda_{j}$ is defined on the generalized zeon algebra $\mathcal{C} \ell_{\mathbf{s}}{ }^{\text {nil }}$ by linear extension of

$$
\Lambda_{j}\left(\nu^{\mathbf{x}}\right):=x_{j} \nu^{\mathbf{x}} .
$$

In particular, for arbitrary multi-exponents $\mathbf{x}, \mathbf{y}$ and scalars $\alpha, \beta$,

$$
\Lambda_{j}\left(\alpha \nu^{\mathbf{x}}+\beta \nu^{\mathbf{y}}\right)=\alpha x_{j} \nu^{\mathbf{x}}+\beta\|\mathbf{y}\|_{1} \nu^{\mathbf{y}} .
$$

Definition 3.5. The dual of the $j^{\text {th }}$ zeon number operator, denoted $\Lambda_{j}{ }^{\star}$, is defined on the generalized zeon algebra $\mathcal{C} \ell_{\mathbf{s}}{ }^{\text {nil }}$ by linear extension of

$$
\Lambda_{j}^{\star}\left(\nu^{\mathbf{x}}\right):= \begin{cases}\left(1 / x_{j}\right) \nu^{\mathbf{x}} & \text { if } x_{j}>0, \\ 0 & \text { otherwise. }\end{cases}
$$

For arbitrary multi-exponents $\mathbf{x}, \mathbf{y}$ and scalars $\alpha, \beta$,

$$
\Lambda_{j}^{\star}\left(\alpha \nu^{\mathbf{x}}+\beta \nu^{\mathbf{y}}\right)=\frac{\alpha}{x_{j}} \nu^{\mathbf{x}}+\frac{\beta}{y_{j}} \nu^{\mathbf{y}} .
$$


An element $u \in \mathcal{C} \ell_{\mathbf{s}}{ }^{\text {nil }}$ is said to be scalar-free if its canonical expansion is of the form

$$
\sum_{\mathbf{x} \neq \mathbf{0}} u_{\mathbf{x}} \nu^{\mathbf{x}}
$$

Let $\mathcal{C} \ell_{\mathbf{s}}{ }^{\text {nil }}$ denote the scalar-free subalgebra of $\mathcal{C} \ell_{\mathbf{s}}{ }^{\text {nil }}$; that is,

$$
\mathcal{C} \ell_{\mathbf{s}}{ }^{\text {nil }}:=\left\{u \in \mathcal{C} \ell_{\mathbf{s}}{ }^{\text {nil }}: u \text { is scalar free }\right\} .
$$

The zeon occupancy operator $\Lambda$ and its dual $\Lambda^{\star}$ are $\Lambda=\bigoplus_{j=1}^{n} \Lambda_{j}$ and $\Lambda^{\star}=$ $\bigoplus_{j=1}^{n} \Lambda_{j}{ }^{\star}$, respectively.

Lemma 3.6. Letting $\mathcal{C} \ell_{\mathbf{s}}{ }^{\text {nil }}$ denote the scalar-free subspace of $\mathcal{C} \ell_{\mathbf{s}}{ }^{\text {iil }}$,

$$
\left.\Lambda \Lambda^{\star}\right|_{\mathcal{C} \ell_{\mathrm{s}}^{\text {nil }}}=\left.\Lambda^{\star} \Lambda\right|_{\mathcal{C} \ell_{\mathrm{s}}^{\text {nil }}}=\mathcal{I}
$$

More specifically, $\Lambda \Lambda^{\star}: \mathcal{C} \ell_{\mathbf{s}}{ }^{\text {nil }} \rightarrow \mathcal{C} \ell_{\mathbf{s}}{ }^{\text {nil }}$ is an orthogonal projection.

Proof. Since the components of multi-exponents are nonnegative integers, writing $\mathbf{x}=\left(x_{1}, \ldots x_{n}\right)$ leads to the component sum as the 1-norm; i.e., $\|\mathbf{x}\|_{1}=$ $\sum_{j=1}^{n} x_{j}$. Let $u=\sum_{\mathbf{x} \neq 0} u_{\mathbf{x}} \nu^{\mathbf{x}} \in \mathcal{C} \ell_{\mathbf{s}}{ }^{\text {nil }}$ and consider

$$
\Lambda\left(\Lambda^{\star} u\right)=\Lambda\left(\sum_{\mathbf{x} \neq 0} \frac{u_{\mathbf{x}}}{\|\mathbf{x}\|_{1}} \nu^{\mathbf{x}}\right)=\sum_{\mathbf{x} \neq 0} \frac{u_{\mathbf{x}}\|\mathbf{x}\|_{1}}{\|\mathbf{x}\|_{1}} \nu^{\mathbf{x}}=u .
$$

A nearly identical argument shows $\Lambda^{\star}(\Lambda u)=u$. From the definitions of $\Lambda$ and $\Lambda^{\star}$, it is apparent that for nonzero scalar $\alpha, \Lambda\left(\alpha \nu^{\mathbf{x}}\right)=0$ if and only if $\mathbf{x}=\mathbf{0}$; i.e., $\alpha \nu^{\mathbf{x}}=\alpha$. The same can be said of $\Lambda^{\star}$; i.e., $\operatorname{ker} \Lambda=\operatorname{ker} \Lambda^{\star}=\mathbb{R}$. As a result, $\operatorname{ker} \Lambda \Lambda^{\star}=\operatorname{ker} \Lambda^{\star} \Lambda=\mathbb{R}$.

Given an $m$-dimensional constraint vector $\mathbf{s}$, a total ordering is induced on the set of multi-exponents $\mathbf{x} \in \mathbb{N}_{0}{ }^{m}$ by defining $\mathbf{x} \preceq \mathbf{y}$ if and only if $\exists k \geq 1$ such that $x_{i} \leq y_{i} \forall i \leq k$. When any such total ordering is assigned to the multi-exponents, one is able to define minimal elements of $\mathcal{C} \ell_{\mathbf{s}}{ }^{\text {nil }}$. This will be useful in subsequent applications in which minimal elements will be associated with optimal solutions.

Definition 3.7. Fixing a total ordering $\preceq$ of the multi-exponents, define a minimal term of $u \in \mathcal{C} \ell_{\mathbf{s}}{ }^{\text {nil }}$ by

$$
\mho u:=u_{\mathbf{x}^{\prime}} \nu^{\mathbf{x}^{\prime}}
$$

where $\mathbf{x}^{\prime} \preceq \mathbf{x}$ for all nonzero multi-exponents in the canonical expansion of $u$. 
The s-zeon algebra will be applied in later sections to sieve out paths satisfying multiple constraints. In order to retain identifying information about the paths themselves, another generalization of zeon algebras is considered.

\subsection{The path algebra $\mathbb{R} \Omega_{n}$}

For fixed positive integer $n$, consider the alphabet $\Sigma_{n}:=\left\{\omega_{i}: 1 \leq i \leq n\right\}$. For convenience, we adopt the following ordered multi-index notation. In particular, letting $\mathbf{u}=\left(u_{1}, \ldots, u_{k}\right)$ for some $k$, the notation $\omega_{\mathbf{u}}$ will be used to denote a sequence (or word) of distinct symbols of the form

$$
\omega_{\mathbf{u}}:=\omega_{u_{1}} \omega_{u_{2}} \cdots \omega_{u_{k}} .
$$

Appending 0 to the set $\Sigma_{n}$, multiplication is defined on the words constructed from elements of $\Sigma_{n}$ by

$$
\omega_{\mathbf{u}} \omega_{\mathbf{v}}= \begin{cases}\omega_{\mathbf{u} . \mathbf{v}} & \text { if } \mathbf{u} \cap \mathbf{v}=\emptyset \\ 0 & \text { otherwise }\end{cases}
$$

where $\mathbf{u} . \mathbf{v}$ denotes sequence concatenation.

One thereby obtains the noncommutative semigroup $\Omega_{n}$, whose elements are the symbol 0 along with all finite words on distinct generators (i.e., finite sequences of distinct symbols from the alphabet $\Sigma_{n}$ ). Since there are only $n$ generators, it is clear that the maximum multi-index size of semigroup elements is $n$. Moreover, these symbols can appear in any order so that the order of the semigroup is $\sum_{k=0}^{n}\left(\begin{array}{l}n \\ k\end{array}\right) k !=\sum_{k=0}^{n}(n)_{k}$.

Defining (vector) addition and real scalar multiplication on the semigroup yields the semigroup algebra $\mathbb{R} \Omega_{n}$ of dimension $\left|\Omega_{n}\right|$. This semigroup algebra will be referred to as a path algebra.

Consider the collection of ordered pairs $P=\left\{\left(\omega_{i}, \omega_{j}\right): i \neq j \in \Omega_{n} \times \Omega_{n}\right.$, and note that $|P|=n^{2}-n$. Imposing an ordering on $P$, a bijection $f: P \rightarrow\left[n^{2}-n\right]$ is obtained. Any $k$-subset of $\left[n^{2}-n\right]$ thereby determines a unique finite word of $\Omega_{n}$ :

$$
\omega_{\mathbf{u}}=\omega_{u_{1}} \cdots \omega_{u_{k+1}} \leftrightarrow\left\{f\left(\left(u_{1}, u_{2}\right)\right), \ldots, f\left(\left(u_{k}, u_{k+1}\right)\right)\right\} .
$$

In this way, one obtains a one-to-one semigroup homomorphism $\phi: \mathcal{C} \ell_{|P|}{ }^{\text {nil }} \rightarrow$ $\Omega_{n}$ onto the words of length two or more in $\Omega_{n}$. Hence, the semigroup algebra $\mathbb{R} \Omega_{n}$ can be regarded as an extension of a zeon algebra.

Remark 3.8. When the pairs of $P$ are unordered, each $k$-subset of $[n(n-1) / 2]$ determines two finite words of $\Omega_{n}: \omega_{\mathbf{u}}$, and its reversion $\widetilde{\omega_{\mathbf{u}}}$; i.e.,

$$
\omega_{\mathbf{u}}=\omega_{u_{1}} \cdots \omega_{u_{k+1}} \leftrightarrow\left\{f\left(\left(u_{1}, u_{2}\right)\right), \ldots, f\left(\left(u_{k}, u_{k+1}\right)\right)\right\} \leftrightarrow \omega_{u_{k+1}} \cdots \omega_{u_{1}}=\widetilde{\omega_{\mathbf{u}}} .
$$




\subsection{Operator calculus on graphs}

As discussed in previous work, the nilpotent adjacency matrix of a finite graph can be used to sieve out the graph's paths and cycles. The entries of this matrix are the null-square generators of a zeon algebra of appropriate dimension for the graph. The null-square properties of the algebra naturally remove entries corresponding to self-intersecting walks from powers of the nilpotent adjacency matrix.

Of interest in the current work is a method of sieving out paths with multidimensional weights (or costs) simultaneously satisfying a number of constraints. Generalized s-zeon generators will be associated with the graph's edges in such a way that paths whose weights exceed the constraints are zeroed out by the algebra's nilpotent properties.

In particular, extending the nilpotent adjacency matrix construction to $\mathcal{C} \ell_{\mathbf{s}}{ }^{\text {nil }} \otimes$ $\mathbb{R} \Omega_{n}$ allows one to enumerate (list) all paths and cycles satisfying multiple constraints in a finite graph by considering powers of the matrix. The associated tree structure underlying the cycle/path enumeration problem is automatically "pruned" by the inherent properties of the algebra.

The first step is defining a nilpotent adjacency matrix that preserves pathidentifying information.

Definition 3.9. Let $G=(V, E)$ be a graph on $n$ vertices, either simple or directed with no multiple edges. Let $\left\{\omega_{i}\right\}, 1 \leq i \leq n$ denote the null-square, noncommutative generators of $\mathbb{R} \Omega_{n}$. Define the path-identifying nilpotent adjacency matrix $\mathcal{A}$ associated with $G$ as the $n \times n$ matrix

$$
\mathcal{A}_{i j}=\left\{\begin{array}{l}
\omega_{j} \text { if }\left(v_{i}, v_{j}\right) \in E, \\
0 \text { otherwise }
\end{array}\right.
$$

Recalling Dirac notation, the $i^{\text {th }}$ row of $\mathcal{A}$ is conveniently denoted by $\left\langle v_{i}\right| \mathcal{A}$ while the $j^{\text {th }}$ column is denoted by $\mathcal{A}\left|v_{j}\right\rangle$. In this way, $\mathcal{A}$ is completely determined by

$$
\left\langle v_{i}|\mathcal{A}| v_{j}\right\rangle= \begin{cases}\omega_{j} & \text { if there exists a directed edge } v_{i} \rightarrow v_{j} \text { in } G \\ 0 & \text { otherwise }\end{cases}
$$

for all vertex pairs $\left(v_{i}, v_{j}\right) \in E$.

Theorem 3.10. Let $\mathcal{A}$ be the path-identifying nilpotent adjacency matrix of an $n$-vertex graph $G$. For any $k>1$ and $1 \leq i \neq j \leq n$,

$$
\omega_{i}\left\langle v_{i}\left|\mathcal{A}^{k}\right| v_{j}\right\rangle=\sum_{k \text {-paths } \mathbf{w}: v_{i} \rightarrow v_{j}} \omega_{\mathbf{w}} .
$$

Moreover,

$$
\left\langle v_{i}\left|\mathcal{A}^{k}\right| v_{i}\right\rangle=\sum_{k \text {-cycles } \mathbf{w} \text { based at } v_{i}} \omega_{\mathbf{w}} .
$$


More specifically, when $i \neq j$, the product of $\omega_{i}$ with the entry in row $i$, column $j$ of $\mathcal{A}^{k}$ is a sum of basis blades indexed by $k$-step paths $v_{i} \rightarrow v_{j}$ in $G$. Moreover, entries along the main diagonal of $\mathcal{A}^{k}$ are sums of basis blades indexed by the graph's $k$-cycles.

Proof. The result follows from straightforward mathematical induction on $k$ using properties of the multiplication in $\mathbb{R} \Omega_{n}$ with the observation that the initial vertex of the walk, $v_{i}$, is unaccounted for in $\left\langle v_{i}\left|\mathcal{A}^{k}\right| v_{j}\right\rangle$, as seen in (3.18) of the matrix definition. Hence, each term of $\left\langle v_{i}\left|\mathcal{A}^{k}\right| v_{j}\right\rangle$ is indexed by the vertex sequence of a $k$-walk from $v_{i}$ to $v_{j}$ with no repeated vertices, except possibly $v_{i}$ at some intermediate step. Left multiplication by $\omega_{i}$ thus sieves out the $k$-paths.

Considering entries along the main diagonal of $\mathcal{A}^{k}$, note that the final step of a $k$-cycle based at $v_{i}$ returns to $v_{i}$ so that left multiplication by $\omega_{i}$ is not required for cycle enumeration.

Note that $\omega_{i}\left\langle v_{i}\right| \mathcal{A}^{k}$ is a row vector whose nonzero entries represent all $k$-paths with initial vertex $v_{i}$. Similarly, $\mathcal{A}\left|v_{j}\right\rangle$ is a column vector whose nonzero entries represent 1-paths with terminal vertex $v_{j}$. Computing the $(k+1)$-paths from $v_{i}$ to $v_{j}$ then requires computing $\omega_{i}\left\langle v_{i}\left|\mathcal{A}^{k} \mathcal{A}\right| v_{j}\right\rangle$. Define the indicator function $\chi_{\left(v_{\ell}, v_{j}\right) \in E}$ by

$$
\chi_{\left(v_{\ell}, v_{j}\right) \in E}= \begin{cases}1 & \text { if }\left(v_{\ell}, v_{j}\right) \in E, \\ 0 & \text { otherwise. }\end{cases}
$$

Letting $m$ denote the number of multiplications involved in this computation,

$$
m=\sum_{\ell=1}^{n} \sharp\left\{k \text {-paths } v_{i} \rightarrow v_{\ell}\right\} \chi_{\left(v_{\ell}, v_{j}\right) \in E} \leq \mid\left\{k \text {-paths with source } v_{i}\right\} \mid \text {. }
$$

It follows immediately that the number of multiplications performed in determining $\omega_{i}\left\langle v_{i}\left|\mathcal{A}^{k}\right| v_{j}\right\rangle$ is bounded above by the number of paths of length at most $k-1$ having initial vertex $v_{i}$. Hence, the next corollary is obtained.

Corollary 3.11. Given a fixed pair of vertices $v_{0}$ and $v_{\infty}$, the complexity of enumerating all $k$-paths from $v_{0}$ to $v_{\infty}$ with the path-identifying nilpotent adjacency matrix is

$$
\left.\mathcal{O}\left(n \mid \text { paths of length } k-1 \text { or less having initial vertex } v_{0}\right\} \mid\right) \text {. }
$$

Remark 3.12. The computational complexity stated above is in terms of basis blade multiplications performed within the algebra. Recall that for disjoint ordered multi-indices $\mathbf{p}, \mathbf{q}$, the product $\omega_{\mathbf{p}} \omega_{\mathbf{q}}=\omega_{\mathbf{p . q}}$ is given by sequence concatenation. Hence, some additional polynomial cost is associated with the implementation of the algebra multiplication. 


\subsection{Operator calculus approach to multi-constrained paths}

The goal now is to extend the path-identifying nilpotent adjacency matrix approach to include weighted edges. In particular, each edge of the graph will be weighted by an $m$-tuple of nonnegative integers. In this manner, paths in the graph will have associated $m$-dimensional additive weights.

Given vectors $\mathbf{x}=\left(x_{1}, \ldots, x_{m}\right)$ and $\mathbf{y}=\left(y_{1}, \ldots, y_{m}\right)$, the notation $\mathbf{x} \leq \mathbf{y}$ is taken to mean the following:

$$
\mathbf{x} \leq \mathbf{y} \Leftrightarrow x_{i} \leq y_{i} \forall i \in\{1, \ldots, m\}
$$

The strict inequality $\mathbf{x}<\mathbf{y}$ is analogously defined.

Given a finite graph $G$ in which each edge is weighted with an $m$-tuple of nonnegative integers and a constraint vector $\mathbf{c}=\left(c_{1}, \ldots, c_{m}\right) \in \mathbb{N}_{0}{ }^{m}$, the multi-constrained path problem is defined as follows.

Definition 3.13. The MCP (Multi-Constrained Path) problem is to find paths p from $v_{0}$ to $v_{\infty}$ in the graph $G$ such that

$$
\mathrm{wt}(\mathbf{p})=\sum_{\left(v_{i}, v_{j}\right) \in \mathbf{p}} \mathrm{wt}\left(\left(v_{i}, v_{j}\right)\right) \leq\left(c_{1}, \ldots, c_{m}\right)=\mathbf{c} .
$$

The goal is to find the set of feasible paths $\mathcal{P}_{f}=\left\{\mathbf{p}=\left(v_{0}, \ldots, v_{\infty}\right): \operatorname{wt}(\mathbf{p})<\mathbf{c}\right\}$, i.e., all paths from $v_{0}$ to $v_{\infty}$ that satisfy multiple constraints simultaneously.

An important variant of the MCP problem that is of particular interest is the associated optimization problem.

Definition 3.14. Letting $\mathcal{P}$ denote the set of feasible paths from $v_{0}$ to $v_{\infty}$ in a weighted graph $G$, the Multi-Constrained Optimal Path problem (MCOP) is to find a path $\mathbf{p}=\left(v_{0}, \ldots, v_{\infty}\right) \in \mathcal{P}$ from $v_{0}$ to $v_{\infty}$ such that

$$
\mathrm{wt}(\mathbf{p}) \leq \operatorname{wt}(\mathbf{q}) \forall \mathbf{q} \in \mathcal{P} .
$$

Note that for fixed $\mathbf{s} \in \mathbb{N}^{m}$, multi-exponents appearing among basis elements $\nu^{\mathbf{x}} \in \mathcal{C} \ell_{\mathbf{s}}{ }^{\text {nil }}$ must satisfy $\mathbf{x}<\mathbf{s}$. Given an $m$-vector $\mathbf{s} \in \mathbb{N}^{m}$, multiplication of arbitrary $\mathbf{s}-z e o n$ blades consequently satisfies

$$
\nu^{\mathbf{x}} \nu^{\mathbf{y}}= \begin{cases}\nu^{\mathbf{x}+\mathbf{y}} & \text { if } \mathbf{x}+\mathbf{y}<\mathbf{s} \\ 0 & \text { otherwise }\end{cases}
$$

In order to apply the OC approach to problems of identifying paths satisfying multiple constraints (represented by $\mathbf{s}$ ), the path-identifying nilpotent adjacency matrix will be extended by allowing entries from the algebra $\mathcal{C} \ell_{\mathbf{s}}{ }^{\text {nil }} \otimes \mathbb{R} \Omega_{n}$. In this approach, a path $\mathbf{u}=\left(u_{1}, \ldots, u_{m}\right)$ of weight $\mathbf{x} \in \mathbb{N}_{0}{ }^{m}$ will be represented in $\mathcal{C} \ell_{\mathbf{s}}{ }^{\text {nil }} \otimes \Omega_{n}$ by an element of the form $\nu^{\mathbf{x}} \omega_{\mathbf{u}}$. The concatenation of this path with another path $\mathbf{v}=\left(v_{1}, \ldots, v_{\ell}\right)$ of weight $\mathbf{y}$ is then represented by the product

$$
\left(\nu^{\mathbf{x}} \omega_{\mathbf{u}}\right)\left(\nu^{\mathbf{y}} \omega_{\mathbf{v}}\right)= \begin{cases}\nu^{\mathbf{x}+\mathbf{y}} \omega_{\mathbf{u} \cdot \mathbf{v}} & \text { if } \mathbf{u} \cdot \mathbf{v} \text { is a path of multi-weight less than } \mathbf{s} \\ 0 & \text { otherwise. }\end{cases}
$$




\subsection{Feasible \& optimal paths in $m$-weighted graphs}

Often in routing problems, there are costs associated with each edge of a graph. Connections between nodes may require time, energy, money, etc. Given an initial vertex $v_{0}$ and terminal vertex $v_{t}$ in a weighted graph, the collection of feasible paths from $v_{0}$ to $v_{t}$ refers to all paths whose associated total costs satisfy some predefined constraints. Among these feasible paths, an optimal path can then be chosen.

Given a vector of constraints $\mathbf{c}=\left(c_{1}, \ldots, c_{m}\right) \in \mathbb{N}^{m}$, properties of the c-zeon algebra $\mathcal{C} \ell_{\mathbf{c}}{ }^{\text {nil }}$ can be used to sieve out the feasible paths from the collection of all paths. The feasible paths can then be ranked and an optimal path chosen.

Definition 3.15. Let $\mathbf{c} \in \mathbb{N}^{m}$, and let $G=(V, E)$ be a graph on $n$ vertices whose edges $\left(v_{i}, v_{j}\right)$ are multi-weighted by vectors $\mathbf{w}_{i j} \in \mathbb{N}_{0}{ }^{m}$. The c-constrained path-identifying nilpotent adjacency matrix associated with $G$ is the $n \times n$ matrix with entries in $\left(\mathcal{C} \ell_{\mathbf{c}}{ }^{\text {nil }}\right)^{m} \otimes \Omega_{n}$ determined by

$$
\Psi_{i j}=\left\{\begin{array}{l}
\nu^{\mathbf{w}_{i j}} \omega_{j} \text { if }\left(v_{i}, v_{j}\right) \in E \\
0 \text { otherwise }
\end{array}\right.
$$

Theorem 3.16. Given a multi-weighted graph $G$ on $n$ vertices with nilpotent multi-weighted adjacency matrix $\Psi$, a vector of constraints $\mathbf{c}=\left(c_{1}, \ldots, c_{k}\right)$, and a pair of distinct vertices $v_{0}$ and $v_{\infty}$, the collection of feasible paths $v_{0} \rightarrow v_{\infty}$ in $G$ is given by

$$
\nu^{\mathbf{0}} \omega_{0} \sum_{\ell=1}^{n}\left\langle v_{0}\left|\Psi^{\ell}\right| v_{\infty}\right\rangle=\sum_{\substack{\text { paths } \\ \text { wt }(\mathbf{p})<\mathbf{c}<}} \nu^{\mathrm{wt}(\mathbf{p})} \omega_{\mathbf{p}}
$$

More specifically, feasible paths exist if and only if $\nu^{\mathbf{0}} \omega_{0} \sum_{\ell=1}^{n}\left\langle v_{0}\left|\Psi^{\ell}\right| v_{\infty}\right\rangle$ is nonzero. For the case $v_{0}=v_{\infty}$, one has

$$
\left\langle v_{0}\left|\Psi^{\ell}\right| v_{0}\right\rangle=\sum_{\substack{\text { cycles } \mathbf{p}: v_{0} \rightarrow v_{0} \\ \operatorname{wt}(\mathbf{p})<\mathbf{c}}} \nu^{\mathrm{wt}(\mathbf{p})} \omega_{\mathbf{p}}
$$

Proof. The result follows from Theorem 3.10 in consideration of combinatorial properties of $\mathcal{C} \ell_{\mathbf{s}}{ }^{\text {nil }}$.

Corollary 3.17. If $\nu^{\mathbf{0}} \omega_{0} \sum_{\ell=1}^{n}\left\langle v_{0}\left|\Psi^{\ell}\right| v_{\infty}\right\rangle \neq 0$, then the optimal path $\mathbf{p}=\left(v_{0}, \ldots, v_{\infty}\right)$ exists and is given by

$$
\mho\left(\nu^{\mathbf{0}} \omega_{0} \sum_{\ell=1}^{n}\left\langle v_{0}\left|\Psi^{\ell}\right| v_{\infty}\right\rangle\right)=\nu^{\mathrm{wt}(\mathbf{p})} \omega_{\mathbf{p}}
$$

Proof. By Theorem 3.16, the collection of all feasible paths $v_{0} \rightarrow v_{\infty}$ is given by $\nu^{\mathbf{0}} \omega_{0} \sum_{\ell=1}^{n}\left\langle v_{0}\left|\Psi^{\ell}\right| v_{\infty}\right\rangle$. By the chosen ordering of paths and definition of $\mho$, the optimal path is as stated. 


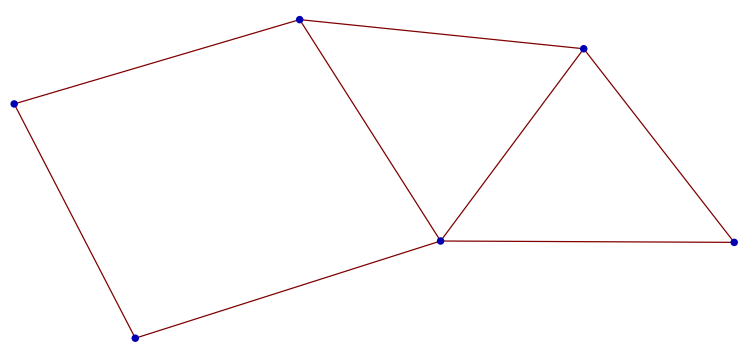

Figure 1: Weighted graph on six vertices.

Example 3.18 (Static case). Figure 1 depicts a weighted graph on six vertices. Each edge is assigned a vector of random positive weights in $\mathbb{N}^{4}$. The graph is not symmetrically weighted; i.e., weights of vertex pairs vary depending on direction. The constraint vector is $\mathbf{c}=(40,27,30,40)$. The weighted pathidentifying adjacency matrix associated with the graph is given by the following:

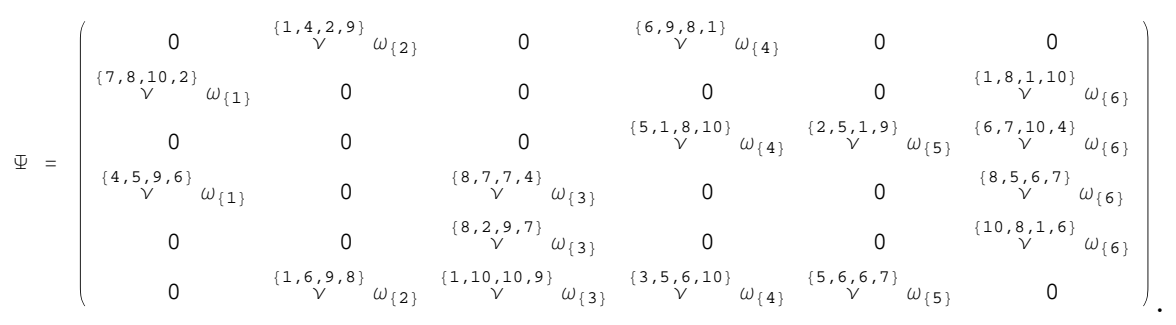

The collection of admissible 4-paths from $v_{1}$ to $v_{3}$ are then computed using Mathematica:

$$
\begin{aligned}
& \text { Four step paths from v1 to v3 satisfying } w \leq\{40,27,30,40\} \\
& \{13,24,16,33\})+\{15,20,18,33\} v^{\prime} \omega_{\{1,2,5,3\}}+\{27,22,29,22\}
\end{aligned}
$$

Let $\mathcal{P}_{f}{ }^{0}$ denote the collection of feasible paths with source $v_{0}$. That is,

$$
\mathcal{P}_{f}^{0}=\left\{\mathbf{p}=\left(v_{0}, \ldots, v_{*}\right): \operatorname{wt}(\mathbf{p})<\mathbf{c}, v_{*} \in V\right\} .
$$

Following the approach of Corollary 3.11, the number of multiplications required in computing $\nu^{\mathbf{0}} \omega_{0}\left\langle v_{0}\left|\Psi^{\ell}\right| v_{\infty}\right\rangle$ is seen to depend on the number of paths of length $\ell-1$ or less having initial vertex $v_{0}$ and simultaneously satisfying the constraints represented by $\mathbf{c}$. The following corollary is obtained as an immediate consequence.

Corollary 3.19. Given a fixed pair of vertices $v_{0}$ and $v_{\infty}$, the complexity of computing the optimal feasible path from $v_{0}$ to $v_{\infty}$ via the operator calculus method is

$$
\mathcal{O}\left(n\left|\mathcal{P}_{f}^{0}\right|\right) \text {. }
$$




\subsection{The dynamic multi-constrained path problem}

Given a finite set $V$ and positive integer $r$, an $r$-dimensional weighting function $\varphi$ is defined on ordered pairs of vertices; i.e., $\varphi: V \times V \rightarrow \mathbb{N}_{0}{ }^{r}$. A weighted graph is then defined by the ordered triple $G=(V, E, \varphi)$, where the weight of an arbitrary edge $\left(v_{i}, v_{j}\right) \in E$ is the $r$-vector $\varphi\left(\left(v_{i}, v_{j}\right)\right)$.

Given a fixed collection of vertices $V$ and fixed weighting function $\varphi: V \times$ $V \rightarrow \mathbb{N}_{0}{ }^{r}$, a weighted graph process on $V$ is defined as a sequence of weighted finite graphs $\left(G_{t}\right):=\left(\left(V, E_{t}, \varphi\right)\right)$. Given a pair of vertices $v_{0}, v_{\infty} \in V$ and a vector $\mathbf{c} \in \mathbb{N}^{r}$ of constraints, the problem being considered is to recover the set of feasible paths $v_{0} \rightarrow v_{\infty}$ simultaneously satisfying the constraints $\mathbf{c}$.

Note that any graph sequence $\left(G_{t}\right)$ naturally induces an associated sequence of weighted path-identifying nilpotent adjacency operators $\left(\Psi_{t}\right)$, where each $\Psi_{t}$ has entries in $\mathcal{C} \ell_{\mathbf{c}}{ }^{\text {nil }} \otimes \Omega_{|V|}$.

It is important to note that a feasible path $\mathbf{p}$ from $v_{0}$ to $v_{\infty}$ is not necessarily unique in the dynamic case because the sequence $\mathbf{p}$ can be partitioned into steps occurring in different frames of the process. As a consequence, scalar coefficients must be introduced to represent the path multiplicity within the collection $\mathcal{P}$.

Theorem 3.20. The collection of feasible paths of length $k \geq 1$ from $v_{0} \rightarrow v_{\infty}$ requiring $m$ or fewer frames is given by

$$
\nu^{\mathbf{0}} \omega_{v_{0}} \sum_{\substack{0 \leq \ell_{1}, \ldots, \ell_{m} \\ \ell_{1}+\ldots+\ell_{m}=k}}\left\langle v_{0}\left|\Psi_{1}^{\ell_{1}} \cdots \Psi_{m}^{\ell_{m}}\right| v_{\infty}\right\rangle=\sum_{k \text {-paths } \mathbf{p}=\left(v_{0}, \ldots, v_{\infty}\right) \in \mathcal{P}} \alpha_{\mathbf{p}} \nu^{\mathrm{wt}(\mathbf{p})} \omega_{\mathbf{p}}
$$

where $\alpha_{\mathbf{p}}$ is a scalar coefficient representing the multiplicity of the path $\mathbf{p}$ in the collection $\mathcal{P}$.

Proof. Note that $k \geq 1$ ensures that at least one of the integers $\ell_{i}$ is nonzero. For fixed nonnegative integers $\ell_{1}, \ell_{2}$ with $\ell_{1}+\ell_{2}=k$,

$$
\begin{aligned}
& \nu^{\mathbf{0}} \omega_{v_{0}}\left\langle v_{0}\left|\Psi_{1}^{\ell_{1}} \Psi_{2}^{\ell_{2}}\right| v_{\infty}\right\rangle=\nu^{\mathbf{0}} \omega_{v_{0}} \sum_{v_{j} \neq v_{0}}\left\langle v_{0}\left|\Psi_{1}^{\ell_{1}}\right| v_{j}\right\rangle\left\langle v_{j}\left|\Psi_{2}^{\ell_{2}}\right| v_{\infty}\right\rangle \\
& =\sum_{v_{j} \neq v_{0}}\left(\sum_{\substack{\ell_{1} \text {-paths } \\
\text { In frame } 1}} \alpha_{\mathbf{p}} \nu^{\operatorname{wt}(\mathbf{p})} \omega_{\mathbf{p}}\right)\left(\sum_{\substack{\ell_{2} \text {-paths } \\
\text { In frame } 2}} \alpha_{\left.\mathbf{q}, v_{j}, \ldots, v_{\infty}\right) \in \mathcal{P}} \nu^{\operatorname{wt}(\mathbf{q})} \omega_{\mathbf{q}}\right) \\
& =\sum_{k \text {-paths }} \sum_{\mathbf{p}=\left(v_{0}, \ldots, v_{\infty}\right) \in \mathcal{P}^{\ell_{1}, \ell_{2}}} \alpha_{\mathbf{p}} \nu^{\mathrm{wt}(\mathbf{p})} \omega_{\mathbf{p}},
\end{aligned}
$$

where $\mathcal{P}^{\ell_{1}, \ell_{2}}$ denotes the collection of feasible paths from $v_{0}$ to $v_{\infty}$ in which $\ell_{1}$ steps occur in frame 1 and $\ell_{2}$ steps occur in frame 2 . Proceeding by induction, the result is established for fixed $m$-tuple of nonnegative integers $\left(\ell_{1}, \ldots, \ell_{m}\right)$ with $\ell_{1}+\cdots+\ell_{m}=k$. Assuming that for positive integer $m_{0}$ and fixed non- 
negative integers $\ell_{1}, \ldots, \ell_{m_{0}}$ with $\ell_{1}+\cdots \ell_{m_{0}}=k^{\prime} \leq k$,

$$
\nu^{0} \omega_{v_{0}}\left\langle v_{0}\left|\Psi_{1}^{\ell_{1}} \cdots \Psi_{m_{0}}^{\ell_{m_{0}}}\right| v_{\infty}\right\rangle=\sum_{k^{\prime} \text {-paths } \mathbf{p}=\left(v_{0}, \ldots, v_{\infty}\right) \in \mathcal{P}^{\ell_{1}, \ldots, \ell_{m_{0}}}} \alpha_{\mathbf{p}} \nu^{\mathrm{wt}(\mathbf{p})} \omega_{\mathbf{p}}
$$

it follows that for $\ell_{m_{0}+1}=k-k^{\prime}$, one has

$$
\begin{aligned}
& \nu^{\mathbf{0}} \omega_{v_{0}}\left\langle v_{0}\left|\Psi_{1}^{\ell_{1}} \cdots \Psi_{m_{0}}^{\ell_{m_{0}}} \Psi_{m_{0}+1}^{\ell_{m_{0}+1}}\right| v_{\infty}\right\rangle \\
& =\nu^{\mathbf{0}} \omega_{v_{0}} \sum_{v_{j} \neq v_{0}}\left\langle v_{0}\left|\Psi_{1}^{\ell_{1}} \cdots \Psi_{m_{0}}^{\ell_{m_{0}}}\right| v_{j}\right\rangle\left\langle v_{j}\left|\Psi_{m_{0}+1}{ }^{\ell_{m_{0}+1}}\right| v_{\infty}\right\rangle \\
& =\sum_{v_{j} \neq v_{0}}\left(\sum_{\substack{k^{\prime} \text {-paths } \\
\text { in }=\left(v_{0}, \ldots, v_{j}\right)}} \alpha_{\mathbf{p}} \nu^{\ell_{1}, \ldots, \ell_{m_{0}}} \text { wt(p) } \omega_{\mathbf{p}}\right)\left(\sum_{\begin{array}{c}
\left(k-k^{\prime}\right) \text {-paths } \mathbf{q}=\left(v_{j}, \ldots, v_{\infty}\right) \\
\text { in } \mathcal{P}^{0, \ldots, \ell_{m}+1}
\end{array}} \alpha_{\mathbf{q}} \nu^{\operatorname{wt}(\mathbf{q})} \omega_{\mathbf{q}}\right) \\
& =\sum_{k \text {-paths } \mathbf{p}=\left(v_{0}, \ldots, v_{\infty}\right) \in \mathcal{P}^{\ell_{1}, \ldots, \ell_{m_{0}+1}}} \alpha_{\mathbf{p}} \nu^{\mathrm{wt}(\mathbf{p})} \omega_{\mathbf{p}},
\end{aligned}
$$

Hence, the result is established for positive integer $m$ :

$$
\nu^{\mathbf{0}} \omega_{v_{0}}\left\langle v_{0}\left|\Psi_{1}^{\ell_{1}} \cdots \Psi_{m}^{\ell_{m}}\right| v_{\infty}\right\rangle=\sum_{k \text {-paths } \mathbf{p}=\left(v_{0}, \ldots, v_{\infty}\right) \in \mathcal{P}^{\ell_{1}, \ldots, \ell_{m}}} \alpha_{\mathbf{p}} \nu^{\mathrm{wt}(\mathbf{p})} \omega_{\mathbf{p}} .
$$

The proof is thus completed by summing over all such $m$-tuples.

Corollary 3.21. The collection of feasible paths of all lengths from initial vertex $v_{0}$ to terminal vertex $v_{\infty} \neq v_{0}$ requiring $m$ or fewer frames is recovered from the canonical expansion of

$$
\nu^{\mathbf{0}} \omega_{v_{0}} \sum_{\substack{0 \leq \ell_{1}, \ldots, \ell_{m} \\ \ell_{1}+\cdots+\ell_{m} \leq n}}\left\langle v_{0}\left|\Psi_{1}^{\ell_{1}} \cdots \Psi_{m}^{\ell_{m}}\right| v_{\infty}\right\rangle=\sum_{\text {paths } \mathbf{p}: v_{0} \rightarrow v_{\infty}} \alpha_{\mathbf{p}} \nu^{\mathrm{wt}(\mathbf{p})} \omega_{\mathbf{p}},
$$

where $\alpha_{\mathbf{p}}$ denotes multiplicity of path $\mathbf{p} \in \mathcal{P}$.

Proof. First, consider the degenerate case $\ell_{i}=0$ for $1 \leq i \leq m$. In this case, the product of $\Psi_{i}$ 's is the identity operator, and $v_{0} \neq v_{\infty}$ gives 0 on the left-hand side of the equation. Observing that the maximum path length is $n=|V|$, the rest follows from Theorem 3.20.

Once the collection of feasible paths is obtained, the optimal path can be selected based on an ordering of the multi-exponents.

Corollary 3.22. Given a preferential ordering of multi-exponents, the optimal path $\mathbf{p}=\left(v_{0}, \ldots, v_{\infty}\right)$ from $v_{0}$ to $v_{\infty}$ in the first $m$ frames of the graph sequence $\left(G_{t}\right)$ is given by

$$
\mho\left(\nu^{\mathbf{0}} \omega_{v_{0}} \sum_{\substack{0 \leq \ell_{1}, \ldots, \ell_{m} \\ 1 \leq \ell_{1}+\ldots+\ell_{m} \leq n}}\left\langle v_{0}\left|\Psi_{1}^{\ell_{1}} \cdots \Psi_{m}^{\ell_{m}}\right| v_{\infty}\right\rangle\right)=\alpha_{\mathbf{p}} \nu^{\mathrm{wt}(\mathbf{p})} \omega_{\mathbf{p}}
$$


provided $\nu^{0} \omega_{v_{0}}\left\langle v_{0}\left|\Psi_{1}^{\ell_{1}} \ldots \Psi_{m}^{\ell_{m}}\right| v_{\infty}\right\rangle \neq 0$ for some $m$-tuple of nonnegative integers $\ell_{1}, \ldots, \ell_{m}$, not all of which are zero. Here, $\alpha_{\mathbf{p}}$ denotes the multiplicity of path $\mathbf{p} \in \mathcal{P}$.

\section{Conclusion}

The operator calculus approach provides convenient symbolic computational tools for a broad range of combinatorial problems and practical applications. The methods developed here can be applied directly to multi-constrained quality of service (QoS) problems as well as problems related to precomputed routing in store-and-forward satellite constellations [3].

\section{References}

[1] R. Bellman, On a routing problem, Quarterly of Applied Mathematics, 16 (1958),87-90.

[2] H.W. Corley, I.D. Moon, Shortest paths in networks with vector weights, Journal of Optimization Theory and Applications, 46 (1985), 79-86. http: //dx.doi.org/10.1007/BF00938761

[3] H. Cruz-Sánchez, R. Schott, Y-Q. Song, G.S. Staples, Operator calculus approach to minimal paths: Precomputed routing in a store-and-forward satellite constellation, Preprint, 2011.

[4] E.W. Dijkstra, A note on two problems in connexion with graphs, $\mathrm{Nu}$ merische Mathematik, 1 (1959), 269271. http://dx.doi.org/10.1007/ BF01386390

[5] L.R. Ford Jr., D.R. Fulkerson, Flows in Networks, Princeton University Press, 1962.

[6] R. Schott, G.S. Staples, Nilpotent adjacency matrices and random graphs, Ars Combinatoria, 98 (2011), 225-239.

[7] R. Schott, G.S. Staples, Nilpotent adjacency matrices, random graphs, and quantum random variables, J. Phys. A: Math. Theor., 41 (2008), 155205.

[8] G.S. Staples, Clifford-algebraic random walks on the hypercube, Advances in Applied Clifford Algebras, 15 (2005), 213-232.

[9] G.S. Staples, A new adjacency matrix for finite graphs, Advances in Applied Clifford Algebras, 18 (2008), 997-991. 